\title{
Eine andere Form der Ungleichheit: Behinderung und soziale Stratifikation in Japans kakusa shakai-Diskussion *
}

\author{
URS MATTHIAS ZACHMANN
}

Abstract

Political discourse on the "society of widening social gaps" (kakusa shakai) in Japan largely focuses on the middle classes. On the one hand, the concept voices the fears of a majority of Japanese citizens of social downgrading and the eventual drop into the new sub-class of "working poor". Neo-liberal politicians, on the other hand, defend social stratification as the natural and eventually desirable outcome of competition, which allows people to "challenge" their current social status and awards perseverance with upward mobility. Both positions, however, seem somewhat self-centered as they largely neglect those who are already at the fringes of Japanese society and, by the unfortunate circumstance of nature or accident, not able to enter the competition on the same terms as the majority. Disability in Japan, albeit less visible than in Western societies, is not a marginal issue even by numbers: 7.2 million people in Japan have some form of physical, mental or psychological disability (as of 2006). Considering that 87 percent of these live with their family, this multiplies the number of those affected by disability in their living circumstances to a sizable percentage of the total population. How then does this social group experience the kakusa shakai? Mindful of the social and demographic changes, the Japanese government since 1990 has initiated a flood of social reforms which, among others, also sought to improve the position of disabled persons by contributing to their equal participation in society. The Services and Supports for Persons with Disabilities Act (Shōgaisha jiritsu shienho) of 2005 constitutes the sum of these endeavors so far, but has right from the start earned the criticism of disability organizations and service providers for being too neo-liberalist and heralding the retreat of the welfare state in disability policies. This article discusses the discourse on disability and social inequality focusing on the Disabilities Act, arguing that especially providers of disability services tend to stress the link between 
disability and social inequality, not only to represent the rights and interests of persons with disabilities, but also in order to fight for their own existence in an increasingly competitive welfare market. Thus, the debate on disability and social inequality is linked with the wider discourse on social inequality, economic competition, and the precariousness of local finances.

Keywords: disability; society of widening social gaps (kakusa shakai); Services and Supports for Persons with Disabilities Act (Shōgaisha jiritsu shien-hō, 2005); normalization; neo-liberalism; welfare system.

\section{Einleitung}

Glück ist das Maß aller Dinge in utilitaristischen Gesellschaftsentwürfen, und das freie, ungehinderte Streben nach demselben das vielleicht einzig legitime Verhalten, welches liberale Varianten dieser Entwürfe gleichermaßen billigen. Mag das Streben des Einzelnen nach Glück auch dem Fortschritt der Gesellschaft insgesamt dienen, so haben sich Individuen - so die Lehre vom Gesellschaftsvertrag - ursprünglich nur der Not gehorchend und zu gegenseitigem Nutzen zu einer Gesellschaft zusammengeschlossen. Gesellschaft als gegenseitiges Leistungsverhältnis setzt jedoch in gewissem Maße Leistungsäquivalenz voraus und das subjektive Vermögen, diese im gesellschaftlichen do ut des auch zu bewirken. Liberale, utilitaristische Entwürfe fordern daher von den ursprünglichen Parteien des Gesellschaftsvertrags, dass sie „frei, gleich und unabhängig“ seien (Nussbaum 2006: 25-35). Selbst eine kontraktualistische Theorie der sozialen Gerechtigkeit, die sich bewusst gegen utilitaristische Vorstellungen ausspricht, beschreibt die Parteien des Gründungsaktes als „voll kooperierende Mitglieder der Gesellschaft über ein vollendetes Leben“, deren Fähigkeiten zudem im „Bereich des Normalen“ liegen (Rawls 1996: 20, 21; Rawls 1971: 25).

Behinderungen im weiteren Sinne - d. h. körperliche oder geistige Beeinträchtigungen, Handlungs- oder Teilnahmebeschränkungen (so die WHO-Definition von disability) - stehen in offensichtlichem Gegensatz zu Gesellschaftsmodellen, die auf Eigennutz und Leistungsäquivalenz setzen. Die Berücksichtigung von Menschen mit Behinderung erfolgt bei diesen Modellen zumeist nur nachgeschaltet und als paternalistischer Reflex auf eine bedauerliche Abweichung von der Norm. Eine Teilhabe bereits in der (rein gedanklichen) Ursituation des Zusammenschlusses und der Festsetzung grundlegender Ordnungsprinzipien einer Gesellschaft erscheint aus dieser Perspektive kaum möglich (Nussbaum 2006: 96-154; Stein 2006).

Der Konflikt um Teilhabe erscheint besonders deutlich in Zeiten verschärften Wettbewerbs und verknappter Ressourcen, wenn von fakti- 
schen Asymmetrien der Gesellschaft vermeintliche Not den sozialen Schleier reißt und eine Verschärfung der Situation die Leistungsäquivalenz und damit die Grundfesten der Gesellschaft zu erschüttern droht. Es ist nicht von ungefähr, dass die japanische Öffentlichkeit - wie auch Gesellschaften andernorts - auf die Kombination von Globalisierungsschub und Wirtschaftskrise regelmäßig mit einem Diskurs der Disparitäten ( $k a k u s a)$ reagiert. Historisch betrachtet reagierten bereits Intellektuelle der Meiji-Zeit auf die Öffnung des Landes und die radikalen Finanzreformen des späten 19. Jahrhunderts mit sozialdarwinistischen Phrasen wie dem „survival of the fittest“ (jakuniku kyōshoku) oder der Disparität von „Gewinnern“ und „Verlierern“ (yüshō reppai) (Nagai 1954). Im neuen Jahrtausend haben diese Phrasen nun ihre Entsprechung in der Rede von einer "Gruppe der Gewinner" (kachigumi) und einer „Gruppe der Verlierer" (makegumi) sowie der Herausbildung einer neuen Klassengesellschaft (Hashimoto 2003: 36-39). Zum Teil wird eine solche tiefgreifende Stratifikation der Gesellschaft als tatsächliches Phänomen aber auch bestritten (vgl. z. B. Hara und Seiyama 2005; anderer Ansicht Tachibanaki 2006). Kakusa fungiert in der politischen Diskussion zumeist auch eher als ideologische Chiffre denn als exakte soziologische Beschreibung. Unbestritten ist jedoch wohl, dass die Wirtschafts- und Strukturreformen während der Amtszeit (2001-2006) von Premierminister Koizumi Jun'ichiro den zentralen politischen Fokus der Diskussion darstellten - eine Diskussion, die sich insbesondere an den neoliberalistischen Ideen entzündete, die den Reformen zugrunde lagen oder ihnen unterstellt wurden (Itoh 2008).

Das im Jahre 2005 verabschiedete „Gesetz zur Unterstützung der Selbständigkeit behinderter Menschen" (Shōgaisha jiritsu shien-hō, hier abgekürzt mit SJSH) zielt auf eine tiefgreifende Neuordnung des Systems der sozialen Sicherung behinderter Menschen in Japan ab. Wie der Titel des Gesetzes bereits andeutet, will das Gesetz behinderten Menschen ermöglichen, ,ein ihren Fähigkeiten und ihrer Eignung angemessenes, selbständiges Leben im Alltag und in der Gesellschaft zu führen" (Art. 1 SJSH). Konkret soll dies zu einer verstärkten Integration behinderter Menschen im regulären Erwerbsleben führen. Darüber hinaus strebt das Gesetz auch eine Hinführung von Menschen mit Behinderung an den „Bereich des Normalen“ an, d. h. an ein Höchstmaß an Selbstbestimmung, aber auch eine damit einhergehende Eigenverantwortlichkeit: So sollen sich behinderte Menschen nun die benötigten Hilfeleistungen und deren Anbieter selbst aussuchen und - unter den Vorzeichen knapper Kassen und verknappter Ressourcen - in gewissem Umfange auch an den entstehenden Kosten beteiligt werden.

Vor dem Hintergrund der Problematik utilitaristischer Gesellschaftsentwürfe und der gesellschaftlichen Stimmung der Zeit ist kaum überra- 
schend, dass auch die Reform der sozialen Sicherung behinderter Menschen seit 2005 mit den Stichworten der Debatte um eine „ungleiche Gesellschaft" (kakusa shakai) geführt wird. Insbesondere Gegner der Reform stellen behinderte Menschen als wehrlose Opfer dar, die - ohnehin schon am Rande der Gesellschaft stehend - nun auf der sich immer schneller drehenden Scheibe des Wettbewerbs von den neoliberalen Reformen als Erste über den Rand gedrückt in den Abgrund der Armut stürzen (vgl. Suzuki 2005; Saito 2007). Eine genauere Untersuchung des Diskurses lässt jedoch erkennen, dass eine so klare Frontziehung wohl kaum möglich ist und der beschworene Geist des Neoliberalismus sich häufig doch als Zerrbild partikularer Interessen entpuppt. Insofern kann auch dieser Teildiskurs als symptomatisch für die allgemeinere Debatte um eine kakusa shakai verstanden werden. Aber auch abgesehen von diesen diskursanalytischen Bezügen bietet die Auseinandersetzung um die Reform der sozialen Sicherung behinderter Menschen Anknüpfungspunkte für andere, strukturell verwandte Debatten um gesellschaftliche Asymmetrien. Hier wären vor allem der Gender-Diskurs sowie der Diskurs um die Rolle von alten Menschen in der zukünftigen Gesellschaft zu nennen. Diese Bezüge bleiben im Übrigen nicht nur abstrakter Art, sondern ergeben sich auch auf ganz alltäglicher Ebene, wenn zum Beispiel die Rolle von Frauen bei der Pflege von behinderten Angehörigen sowie die Problematik der altersbedingten Behinderungen berücksichtigt werden (Nussbaum 2006: 100-101; Motozawa 2009; Traphagan 2007).

Die folgende Untersuchung gibt zunächst eine Übersicht über die Situation behinderter Menschen und das System der sozialen Sicherung in Japan, stellt sodann den wesentlichen Regelungsinhalt der Reform von 2005 vor und beleuchtet schließlich in einer Diskursanalyse die aus Anlass der Reform geführte Debatte um Behinderung und soziale Stratifikation in Japan nach 2005, um so eine differenziertere Bewertung der geführten Debatte im Lichte der eingangs skizzierten Problematik von Behinderung, Freiheit und Leistungsäquivalenz zu ermöglichen.

Der Fokus dieser Untersuchung liegt dabei auf der Analyse des Diskurses um die japanische Behindertenpolitik ab 2005 im Lichte der kakusa shakai-Debatte. Die nachfolgende Darstellung der Situation behinderter Menschen sowie der Behindertenpolitik in Japan erfolgt im Hinblick auf diese Analyse und ist daher notwendigerweise eingeschränkt. Ebenso sei angemerkt, dass sich die in Japan geführte Debatte - trotz ihrer lokalen Charakteristika - selbstverständlich in einen globalen Diskurs um die Stärkung der Rechte von Menschen mit Behinderung bei gleichzeitigem Rückzug des Wohlfahrtsstaates einbettet, einen Diskurs, der seinen jüngsten Höhepunkt in der Auseinandersetzung um die neue UN-Konvention von 2006 (in Kraft seit 2008) über die Rechte von Menschen mit Behinderung gefunden hat. ${ }^{1}$ Da gerade auch der im 
Folgenden untersuchte Fall Japans zeigt, wie komplex das Geflecht von Interessen und Motivationen ist, die diese Debatte umgeben und durchdringen, würde ein aussagekräftiger internationaler Vergleich den Rahmen dieser Untersuchung bei Weitem übersteigen.

\section{Definition von Behinderung und demographische Entwicklung in Japan}

Seit 1993 enthält das Grundlagengesetz für Menschen mit Behinderungen (Shōgaisha kihon-hō) von 1970 eine für Menschen mit körperlicher oder geistiger Behinderung einheitlich geltende Definition. ${ }^{2}$ Artikel 2 des Gesetzes definiert Menschen mit Behinderung (shōgaisha) als „Personen, die aufgrund ihrer körperlichen, geistigen oder psychischen Behinderung auf Dauer wesentliche Beschränkungen im Alltag sowie im gesellschaftlichen Leben erfahren“. Diese allgemeine Definition lehnt sich zwar an internationale Vorbilder an, insbesondere an den US-amerikanischen Americans with Disabilities Act (ADA) von 1990 (Heyer 1999: 108), allerdings sei darauf hingewiesen, dass die konkrete Ausfüllung des Begriffes eine eigenständige ist und nicht einheitlich erfolgt, sondern in den zahlreichen Einzelgesetzen, die sich mit der sozialen Sicherheit behinderter Menschen befassen, jeweils verschieden erfolgt (vgl. hierzu Takizawa 2006: 15-54, 219-244). Ungeachtet dieser Ausdifferenzierung im Detail folgen diese Gesetze der allgemeinen Tendenz des Grundlagengesetzes in der expliziten Bezugnahme auf die soziale Situation, in der sich ein Mensch aufgrund seiner Behinderung in der Gesellschaft befindet. Das Grundlagengesetz richtet sich damit bewusst gegen die traditionelle medizinische Definition von Behinderung, die sich in der isolierten Feststellung der körperlichen, geistigen oder psychischen Abweichung von der „Norm“ erschöpfte. Die Hinwendung zu einem das rein medizinische Verständnis übersteigenden sozialorientierten Begriff von Behinderung, die in den 1980ern stattfand, hat weitreichende Folgen für die Integration von Menschen mit Behinderung in der Gesellschaft (Finkelstein 1980).

Aufgrund der internationalen Definitionsunterschiede sind Vergleiche nur von begrenztem Aussagewert. Allgemein gilt jedoch, dass die Zahl der in Japan offiziell als Menschen mit Behinderung anerkannten Personen mit ca. 7,2 Mio., d. h. einem Anteil von ca. 5,6 Prozent der Gesamtbevölkerung (Stand 2005/6) relativ niedrig ist (Naikaku-fu 2009: 5). Im Vergleich dazu liegt in Deutschland der Anteil der schwerbehinderten Menschen mit 6,7 Mio. deutlich höher bei 8,4 Prozent der Gesamtbevölkerung, während für Gesamteuropa der Anteil von Menschen mit Behinderung sogar auf ca. 15 Prozent der Gesamtbevölkerung geschätzt wird. ${ }^{3}$ Diese Differenz liegt vor allem in der sehr restriktiven Auslegung von 
Behinderung durch den japanischen Gesetzgeber begründet (Heyer 1999: 111). Bedenkt man jedoch, dass in Japan Menschen mit Behinderung oft stärker auf die Unterstützung von Familienmitgliedern angewiesen sind und dass der Anteil derer, die nicht in stationären Einrichtungen, sondern zu Hause leben, mit 6,3 Mio. Personen - was 87 Prozent aller Menschen mit Behinderung (Naikaku-fu 2009: 5; Motozawa 2009: 400) entspricht - besonders hoch liegt, so ist die Zahl derer, die in Japan direkt oder indirekt mit den Problemen der Behinderung im Alltag konfrontiert werden, weitaus höher, als es die soeben genannten statistischen Werte unmittelbar anzeigen.

Wie auch in Europa gilt in Japan die größte demographische Sorge der Zunahme der Zahl von Menschen mit Behinderung durch die sogenannte „Überalterung der Gesellschaft“ ${ }^{4}$ Dies gilt für Japan umso mehr, als das Phänomen der Überalterung hier bekanntlich deutlicher und stärker ausgeprägt ist als in anderen Industrienationen (vgl. Coulmas et al. 2008). Die Kombination von demographischem Wandel und einer altersbedingten Anfälligkeit für bestimmte Behinderungen hat in Japan dazu geführt, dass zwischen 1960 und 2006 der Anteil der über 65-Jährigen mit körperlicher Behinderung von 31,4 Prozent auf 61,8 Prozent der Gesamtzahl aller Personen mit körperlichen Behinderungen gestiegen ist und sich damit verdoppelt hat. Und während der Anteil der Behinderten unter 65 Jahren seit 1987 rückläufig ist, ist der Anstieg der absoluten Zahl seit dieser Zeit um etwa eine Million einzig in dem Alterssegment der über 65-Jährigen zu verbuchen (Naikaku-fu 2009: Anhang, Tabelle 2-2).

Der rasante Anstieg des Anteils von Menschen mit Behinderung in dieser Altersgruppe, dessen ungebremstes Wachstum aufgrund der allgemeinen demographischen Entwicklung auch in der Zukunft zu erwarten ist, stellt die soziale Absicherung von Menschen mit Behinderung gegenwärtig vor Herausforderungen, mit denen die allgemeinen Sozialversicherungssysteme in Japan bereits früher konfrontiert wurden - und sich auch gegenwärtig noch konfrontiert sehen (Conrad 2001; Arai 2002). Zur gleichen Zeit jedoch, als die demographische Entwicklung der Überalterung sich auch in der Zunahme von älteren Menschen mit Behinderung allmählich abzeichnete, d.h. in den späten 1980er Jahren, nahm eine andere Entwicklung im Bereich der Behindertenpolitik ihren Ausgang. Als deren Ergebnis ist der bereits angesprochene Paradigmenwechsel zu sehen, namentlich der Wechsel von einem medizinischen Modell hin zu einem sozialen Modell der Behinderung und damit der Betonung von Gleichberechtigung und sozialer Teilhabe. Bemerkenswerterweise steht die demographische Entwicklung dem programmatischen Wechsel nicht entgegen, sondern unterstreicht dessen Dringlichkeit noch. In einer ironischen Volte führt diese Verstärkung jedoch gleichzeitig zu einer 
Neuausrichtung der gegenwärtigen politischen Debatte und der teilweisen Abkehr bestimmter Interessengruppen von den Prinzipien des sozialen Modells.

\section{Der Paradigmenwechsel der japanischen Behindertenpolitik seit den 1980ern}

Zeitgeschichtliche Darstellungen der Entwicklung der japanischen Behindertenpolitik sehen den Impuls für einen Paradigmenwechsel sowohl in internationalen Kampagnen als auch dem wachsenden Bewusstsein für Missstände im Inland begründet (Arai 2003: 304-313; Heyer 1999: 110-122). Zudem ist der Paradigmenwechsel im Kontext der Veränderung der Sozialversicherungssysteme im Ganzen zu verstehen.

An internationalen Impulsen wird insbesondere das von den Vereinten Nationen im Jahre 1981 ausgerufene „Internationale Jahr der Menschen mit Behinderung" hervorgehoben, das zum ersten Mal den Begriff der „Normalisierung" als Programm sowie Gleichberechtigung und volle gesellschaftliche Teilhabe als Ziel einer zukunftsorientierten Behindertenpolitik offiziell auf breiter Ebene propagierte (Heyer 1999: 106-107; Heyer 2000). Normalisierung im Kontext von Behinderung hat sowohl einen sozialen wie auch individuellen Aspekt: Das Konzept zielt einerseits auf die Erschaffung einer Gesellschaft ab, in der Menschen mit Behinderung die gleichen Möglichkeiten der Teilhabe am gesellschaftlichen Leben haben wie Menschen ohne Behinderung, insbesondere bei der Beschäftigung (Arai 2003: 304-305). Der individuelle Anteil hingegen richtet sich nach den Vorstellungen seiner Pioniere darauf, die Lebensverhältnisse von Menschen mit Behinderung so weit als möglich denjenigen von Menschen ohne Behinderung anzugleichen (vgl. Wolfensberger 1972). ${ }^{5}$ Die Vereinten Nationen propagierten dieses Konzept weiterhin in einer „Internationalen Dekade der Menschen mit Behinderung“ (19831992), an die sich wiederum eine UN Decade of Disabled Persons in Asia (1993-2002) anschloss.

So intuitiv das Konzept der Normalisierung erscheint, stand es doch im Gegensatz zur in Japan (wie auch in den westlichen Industrienationen) bis in die 1980er Jahre hinein im Bereich der Behindertenpolitik und der Sozialpolitik allgemein vorherrschenden Philosophie des „Wohlfahrtsstaates" und des Individualschutzes (Arai 2003: 307). Dieser Philosophie lag das Bild der klassischen Leistungsverwaltung zugrunde, die sich in einem strengen Subordinationsverhältnis um das Wohl der einzelnen Bürger kümmerte und durch hoheitliche Maßnahmen deren Schutz besorgte. Im Bereich der sozialen Sicherheit für Menschen mit Behinderung bedeutete dies insbesondere, den „speziellen Bedürfnissen“ (special needs, auf Japanisch: $n \bar{\imath} z u$ ) von Menschen mit Behinderungen Rechnung 
zu tragen - und dies typischerweise in eigens dafür bestimmten Einrichtungen (Heimen, Werkstätten etc.). Es liegt nahe, dass dieser auf hoheitlichen Maßnahmen (sochi) basierende Ansatz tendenziell die „Besonderheit" von Menschen mit Behinderung betont und eine Differenz zur „normalen Gesellschaft" erzeugt, die sich auch räumlich in der Abgeschlossenheit von stationären Einrichtungen zeigt. Skandalöse Misshandlungsfälle, insbesondere die im Jahre 1984 in einem psychiatrischen Krankenhaus in der Stadt Utsunomiya aufgedeckten Fälle (vgl. Heyer 1999: 118) zeigten, dass diese Abgeschlossenheit nicht nur einen besonderen "Schutz" bot, sondern auch besondere Gefahren in sich bergen konnte.

Die japanische Regierung reagierte auf die internationalen Impulse und aufgedeckten Mängel mit einer sukzessiven, bis in die jüngste Zeit hineinreichenden grundlegenden Reform des sozialen Sicherungssystems für behinderte Menschen im Sinne des beschriebenen Normalisierungskonzeptes. Bereits 1984 erfolgte eine Revision des Wohlfahrtsgesetzes von 1949 für körperlich behinderte Menschen (Shintai shōgaisha fukushi$h \bar{o})$. Weitere wichtige Stationen waren - neben zahlreichen anderen Reformmaßnahmen, die jedoch hier nicht im Einzelnen ausgeführt werden können - die Revisionen der acht Wohlfahrtsgesetze im Jahr 1990, die des Grundlagengesetzes in den Jahren 1994 und 2004 sowie zwei Aktionspläne für Menschen mit Behinderung für die Fiskaljahre 1995-2002 und 2003-2012 (hierzu Arai 2009: 390-393). Begleitet wurden diese Reformen durch Aufklärungsarbeit in der Öffentlichkeit, deren Impulse auch von populären Medien aufgenommen wurden. ${ }^{6}$

Die genannten Reformen suchten den beabsichtigten Normalisierungsprozess durch zahlreiche konkrete Maßnahmen herbeizuführen, von denen hier nur die wichtigsten und folgenreichsten genannt werden sollen: So sind die Kommunen nun zwingend verpflichtet, verbindliche Pläne mit konkreten Zielvorgaben für das Leben von Menschen mit Behinderungen in der Region zu erstellen. Damit geht die Übertragung der Verwaltungszuständigkeit für die Behindertenfürsorge von den Präfekturen auf die lokalen Verwaltungsbehörden großer Städte einher. Zudem werden seit 1990 häusliche Pflegedienste explizit als Pflegesystem anerkannt und ihre Ausweitung gegenüber den institutionellen Pflegeeinrichtungen noch angestrebt. Zu diesem Zweck sind nun auch private Unternehmen als Anbieter von häuslichen Pflegedienstleistungen zugelassen. Die für den einzelnen Menschen mit Behinderung wohl einschneidendste Veränderung liegt jedoch in der im Jahre 2003 erfolgten Einführung des Systems zur Deckung häuslicher Pflegekosten (shien-pi seido) (siehe z. B. Arai 2003: 318-319; Motozawa 2009: 408). Anders als das traditionelle maßnahmenbasierte System ordnet nun nicht mehr die Behörde die erforderlichen Pflegeleistungen an; vielmehr schließt der Empfänger der 
Leistungen nun selbst Verträge mit einem Dienstleister seiner Wahl ab und ist auch unmittelbarer Schuldner des dafür zu entrichtenden Entgelts. Die zuständige Behörde genehmigt lediglich die bestellten Pflegeleistungen und stellt dem Empfänger den dafür erforderlichen Geldbetrag zur Verfügung (ähnlich dem ,,persönlichen Budget“ für Menschen mit Behinderung in Deutschland). Allerdings wird zugleich ein Eigenanteil erhoben, der jedoch je nach den finanziellen Möglichkeiten des Empfängers bis auf Null abgesenkt werden kann.

Der Paradigmenwechsel von „Schutz“ zu „Unterstützung“, wie er in dem Systemwechsel von institutioneller Pflege zu gemeinschaftsbasierter Pflege sowie der Umstellung von einem maßnahmenbasierten zu einem leistungsempfängerorientierten System zum Ausdruck kommt, dient vorderhand der Normalisierung, d. h. der Förderung eines selbstbestimmten Lebens behinderter Menschen in einer offenen Gesellschaft. Gleichzeitig ist diese Entwicklung jedoch auch vor dem Hintergrund des Umbaus der Sozialsysteme in Zeiten demographischen Wandels und wirtschaftlichen Nullwachstums zu sehen (Conrad 2001; Arai 2002). Insbesondere im Vergleich zu dem 1997 etablierten Pflegeversicherungssystem ergeben sich Parallelen, wie etwa die Einbeziehung mehrerer Dienstleister oder die Erhebung eines Eigenbetrages, die man unter dem Stichwort der „Ökonomisierung in Zeiten verknappter Ressourcen“ zusammenfassen könnte. Dieser Aspekt wird im Zusammenhang mit dem Gesetz zur Unterstützung der Selbständigkeit behinderter Menschen von 2005 zu diskutieren sein.

\section{Das Gesetz zur Unterstützung der Selbständigkeit behinderter Menschen}

Das im November 2005 erlassene „Gesetz zur Unterstützung der Selb-

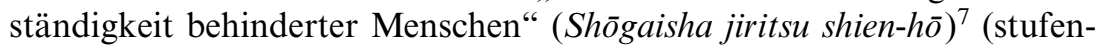
weise in Kraft seit April bzw. Oktober 2006) kann als summa der bereits beschriebenen Maßnahmen verstanden werden, insofern es diese in einen geordneten und systematischen Zusammenhang setzt und weitere Mängel zu beheben sucht. Als neuer Grundpfeiler der Behindertenfürsorge steht es im Mittelpunkt der Debatte um Behinderung und soziale Disparität.

Unmittelbarer Anlass für den Erlass des Gesetzes war die Kritik an bestimmten Schwachstellen des bisherigen Fürsorgesystems, namentlich: (1) die gesonderte Behandlung von Menschen mit körperlicher, geistiger und psychischer Behinderung; (2) der bisherige Ausschluss von Menschen mit psychischen Störungen aus dem System zur Deckung häuslicher Pflegekosten; (3) die unzureichende Bereitstellung von Leistungen in manchen (insbesondere ländlichen) Kommunen; (4) die mangelnde 
Förderung und Unterstützung von behinderten Menschen bei der Aufnahme einer Arbeit; und (5) die mangelnde Transparenz des Bewilligungsverfahrens. ${ }^{8}$ Allerdings spielte wohl auch die sich mit dem System zur Deckung häuslicher Pflegekosten abzeichnende Kostenexplosion eine Rolle. So stieg nach Angaben des Ministeriums für Gesundheit, Arbeit und Wohlfahrt (Kōseirōōoshō, im Folgenden abgekürzt als MHLW) die Zahl derjenigen, die häusliche Pflegedienste in Anspruch nahmen, zwischen April 2003 und Oktober 2004, also binnen anderthalb Jahren, um mehr als die Hälfte an. Entsprechend stieg auch das für diese Pflegedienste erforderliche Budget sprunghaft an. Das für 2005 anvisierte Budget lag beispielsweise bereits 54 Prozent über dem Budget von 2002, bevor das neue System eingeführt wurde (MHLW 2005).

Das Gesetz zur Förderung der Selbständigkeit behinderter Menschen von 2005 reagierte auf die beanstandeten Mängel durch entsprechende Zielsetzungen. So richtet sich das Gesetz nicht nur auf die Erschaffung eines einheitlichen Fürsorgesystems für alle Menschen mit Behinderung in der Hand eines einzigen Verwaltungsträgers (der Städte und Kommunen), sondern auch auf die verstärkte Unterstützung von behinderten Menschen bei der Eingliederung in das normale Erwerbsleben. Des Weiteren zielt es ab auf eine (1) „Regulierungsharmonisierung“ zur Aktivierung der beschränkten regionalen Ressourcen im Einsatz für behinderte Menschen, sieht die (2) Verbesserung der Transparenz und Präzisierung des Bewilligungsverfahrens sowie der zugrundeliegenden Standards vor und plant schließlich die (3) Einführung eines Schemas zur gerechten Lastenverteilung der anfallenden Kosten, wobei auch ein Eigenanteil für den Empfänger vorgesehen ist.

Die konkrete Realisierung der Zielsetzungen des Gesetzes soll hier nicht weiter ausgeführt werden. Für die folgende Diskussion sollen lediglich zwei Beispiele angesprochen werden: Zur Förderung der Eingliederung von Behinderten in das reguläre Erwerbsleben schafft das Gesetz das alte System von Behindertenwerkstätten (fukushi kōjō) und Beschäftigungseinrichtungen (jusan shisetsu) ab und setzt an deren Stelle die Kombination von Betrieben zur Unterstützung des Übergangs in das Erwerbsleben (shürō ikō shien jigyō) und Betrieben zur Unterstützung andauernder Erwerbstätigkeit (shūrō keizoku shien jigyō). Erstere sollen behinderte Menschen durch Ausbildungsmaßnahmen innerhalb von 2 Jahren auf das Erwerbsleben vorbereiten (Art. 5 Abs. 14 SJSH); letztere sollen behinderten Menschen, die noch keine reguläre Arbeit gefunden haben, die Möglichkeit der Beschäftigung und Weiterqualifikation bieten (Art. 5 Abs. 15 SJSH). Dabei wird zwischen Einrichtungen unterschieden, bei denen der behinderte Mensch in einem Anstellungsverhältnis steht (Typ A) oder ohne einen Arbeitsvertrag beschäftigt ist (Typ B). Je nach Beschäftigungsform steht dem behinderten Menschen eine Arbeits- 
vergütung $(k \bar{o} c h i n)$ in unterschiedlicher Höhe zu. Im Rahmen des im Jahre 2007 verabschiedeten Fünfjahresplans zur Förderung des Programms „Von der Wohlfahrt zur Beschäftigung“ (Fukushi kara koyōe) beabsichtigt die Regierung, den bisherigen Anteil von behinderten Menschen im regulären Erwerbsleben zu steigern (vgl. MHLW 2007a).

Als zweites Beispiel sei die Einführung einer „gerechten Lastenverteilung" angesprochen, die für den Einzelnen konkret in einer Selbsttragungspflicht (für Essen und anderes) in den Einrichtungen sowie einer angemessenen Beteiligung an den Kosten der beanspruchten Pflegeleistungen resultiert. Wie auch bei der Pflegeversicherung von 1997 wird ein regulärer Eigenanteil von 10 Prozent als angemessen erachtet, jedoch kann dieser entsprechend den finanziellen Möglichkeiten des Empfängers abgesenkt werden. Tatsächlich bedeutet dies die Anwendung des Prinzips der nutzungsproportionalen Eigenbeteiligung (ōeki futan), während das Prinzip der Eigenbeteiligung gemäß Leistungsfähigkeit (ōnō $f u$ tan) nur nachrangig Anwendung findet. Die Kritik der Interessenverbände konzentriert sich vor allem auf diesen Aspekt des Eigenanteils.

\section{Die wirtschaftliche und soziale Lage von Menschen mit Behinderung in Japan}

Bevor wir uns der Diskussion des Selbständigkeitsgesetzes von 2005 im Lichte der Debatte sozialer Disparitäten (kakusa) zuwenden, empfiehlt sich ein kurzer Blick auf die tatsächliche wirtschaftliche und soziale Lage von Menschen mit Behinderung in Japan. Diese zeigt selbst an den grundlegendsten Indikatoren, namentlich Einkommen und Beschäftigung, dass bereits bei Einführung des Gesetzes eine erhebliche Varianz unter behinderten Menschen bestand und noch besteht, sodass von einheitlichen sozialen Verhältnissen nur im allergröbsten Maßstab gesprochen werden kann.

Die Hauptversorgungsquellen für Menschen mit Behinderung sind die Behindertenrente (shōgai nenkin) sowie der Lohn, den sie in den jeweiligen Beschäftigungsformen erhalten. Die Behindertengrundrente (shōgai kiso nenkin) für Menschen mit einer Behinderung 2. Grades betrug im Jahr 2009 monatlich JPY 66008 und für Menschen mit einer Behinderung 1. Grades JPY 82508 (Naikaku-fu 2009: 106). Behinderte Menschen, die zudem eine Sozialrente erhielten (ca. 5 Prozent von ihnen), bezogen im Jahr 2007 eine Gesamtrente von durchschnittlich JPY 105595 (Shakai Hoken-cho 2009: 13-14). Hinzu kommen Zuschläge für Ehepartner und Kinder sowie verschiedenartige Sonderzuwendungen in besonderen Fällen. Über die Behindertengrundrente hinaus besteht daher bereits in der staatlichen Zuwendung eine erhebliche individuelle Varianz. Der Hauptdifferenzierungsfaktor liegt jedoch in der Beschäftigung. 
Die Beschäftigung von Menschen mit Behinderung erfolgt in zahlreichen Formen, wobei die Beschäftigung in regulären Arbeitsverhältnissen sowie in Behinderteneinrichtungen die häufigste ist. Die Beschäftigungsquote in regulären Arbeitsverhältnissen ist trotz der gesetzlich vorgeschriebenen Mindestquoten zwar noch gering, aber von steigender Tendenz. So waren im Jahre 2008 in privatwirtschaftlichen Unternehmen ca. 326000 Menschen mit Behinderung beschäftigt (das sind 7,6 Prozent mehr als im Vorjahr), im öffentlichen Dienst ca. 47000 Personen und in juristischen Personen des öffentlichen Rechts ca. 5000 Personen - also insgesamt nur 5 Prozent der ca. 7,2 Mio. Menschen mit Behinderung in Japan (Naikaku-fu 2009: 59-61). Diese Beschäftigung wird zum Teil erheblich von staatlicher Seite subventioniert. Allerdings ist ein deutlicher positiver Trend zu verzeichnen: So steigt die Beschäftigungsquote nicht nur in privatwirtschaftlichen Unternehmen, auch die staatlichen Arbeitsvermittlungen melden einen Anstieg sowohl der Arbeitssuchenden als auch der erfolgreich vermittelten Fälle (Naikaku-fu 2009: 68).

Eine andere Form der Beschäftigung findet in den bereits beschriebenen Werkstätten und Beschäftigungszentren statt. Hier liegt die Arbeitsvergütung $(k \bar{o} c h i n)$ in Betrieben zur Unterstützung andauernder Erwerbstätigkeit des Typs A sowie Behindertenwerkstätten des alten Systems durchschnittlich bei monatlich ca. JPY 101000 bzw. 118 000. Bei Betrieben des Typs B und den Beschäftigungseinrichtungen alten Stils (jusan) liegt sie bei jeweils knapp JPY 12000 pro Monat (MHLW 2007b).

Die meisten behinderten Menschen, die in irgendeiner Form werktätig sind, stammen aus der Gruppe der Menschen mit körperlicher Behinderung (zugleich mit ca. 3,5 Mio. die größte Gruppe). Gemäß einer Untersuchung des MHLW lag hier die Beschäftigungsquote im Jahre 2006 bei 20,4 Prozent (Beschäftigung in Behinderteneinrichtungen eingerechnet). Nach Arbeitgebern unterschieden stellten unter diesen die in einem regulären Beschäftigungsverhältnis befindlichen Personen mit 34,9 Prozent die größte Gruppe. Die Höhe des Verdienstes schwankt jedoch auch hier stark. Allenfalls lässt sich ein Schwerpunkt im Verdienstsegment zwischen JPY 70000 und 110000 erkennen (MHLW 2008a: 42).

Bereits ein kursorischer Blick zeigt, dass die Gruppe von behinderten Menschen in der japanischen Gesellschaft wirtschaftlich keine homogene ist; vielmehr besteht eine erhebliche Varianz in den Einkommensverhältnissen. Betrachtet man die Einkommensverhältnisse von behinderten Menschen isoliert, wäre die Mehrheit zwar durchaus am untersten Rand des Spektrums anzusiedeln, allerdings ist die familiäre Einbindung von behinderten Menschen in Japan im Vergleich zu anderen Industrienationen besonders eng (Motozawa 2009; Aoki 2006: 26), sodass hier die tatsächlichen wirtschaftlichen Verhältnisse an die Verteilung in der gesamten Gesellschaft gekoppelt zu sehen sind. Insofern nehmen Menschen 
mit Behinderung an der allgemeinen sozialen Disparität teil. Darüber hinaus erscheint Beschäftigung als der individuelle Schlüssel zu sozialer Differenzierung und macht zumindest bei einer kleinen, aber wachsenden Gruppe von behinderten Menschen den tatsächlichen Unterschied von wirtschaftlicher Selbständigkeit oder Abhängigkeit aus.

Die noch relativ geringe Beschäftigungsquote von maximal 20,4 Prozent bei körperlich Behinderten zeigt jedoch auch, dass die soziale Situation der meisten Behinderten von relativer Ausgegrenztheit geprägt und es bis zum Ziel einer von Normalisierung geprägten „kohärenten Gesellschaft" (kyōsei shakai) noch ein weiter Weg ist. ${ }^{9}$ Dies entspricht laut einer Umfrage des Kabinett-Büros im Jahre 2007 auch dem Bewusstsein der Mehrheit der Japaner, die zu 85 Prozent das Ziel der „,kohärenten Gesellschaft" bejahten. Eine ebenso große Mehrheit erklärte jedoch, dass nach wie vor Diskriminierungen und Vorurteile gegenüber Behinderten bestünden. Eine leichte Mehrheit der Befragten (61,2 Prozent) sah zugleich einen Fortschritt der Maßnahmen zur Verbesserung der Situation im Vergleich zu fünf Jahren zuvor (Naikaku-fu 2007: 18-21). Behindertenverbände und Interessenverbände der Behindertenfürsorge hingegen schätzen die Situation anders ein, wie die im Folgenden beschriebene Diskussion zeigen wird.

\section{Die Diskussion um Behinderung und soziale Disparität (kakusa shakai)}

Die japanischen Behindertenverbände haben sich insgesamt den verschiedenen vorherrschenden Paradigmen der Behindertenpolitik angepasst. Orientierte sich die ältere Generation noch an dem Wohlfahrtsparadigma, dem Prinzip des Individualschutzes und der Geltendmachung von „speziellen Bedürfnissen“ (nīzu), so konzentrierte sich die jüngere Generation im Einklang mit dem allgemeinen Paradigmenwechsel zunehmend auf die Durchsetzung von universellen Rechten für Behinderte, insbesondere des Rechts auf gesellschaftliche Teilhabe, Selbstbestimmung und Integration in allen Bereich der Gesellschaft (Heyer 1999: 122-128). Eine qualitative Analyse des Diskurses um Behinderung und soziale Disparität legt jedoch nahe, dass die demographische und wirtschaftliche Entwicklung seit 2005 das Pendel wieder zurückschlagen lässt. So wenden sich Behindertenverbände, vor allem aber Interessenverbände der Behindertenfürsorge aus Protest gegen vorgebliche neoliberale Tendenzen in der Behindertenpolitik zunehmend wieder dem früheren Paradigma von Schutz und Wohlfahrt zu, dies aber nicht ohne wie im Folgenden deutlich wird - neben den Interessen von behinderten Menschen auch eigene Interessen zu verfolgen. 
Zunächst ist festzuhalten, dass die Diskussion um Behinderung und soziale Disparität - außer in wenigen spektakulären Fällen - kaum breite mediale Aufmerksamkeit erhält (Tada 2007: 15; Saito 2007: 7) und zumeist abseits davon in Publikationen geführt wird, die sich an behinderte Menschen und Vertreter der Behindertenfürsorge richten oder an Vertreter von Minderheitenrecht, wie zum Beispiel die Zeitschrift Minna no Negai der Japanese Association on Disability and Difficulty (Zenkoku Shōgaisha Mondai Kenkyūkai) oder Publikationen der Shōgaisha Seikatsu Shien Shisutemu Kenkyūkai [Arbeitsgemeinschaft für ein System zur Unterstützung des Alltagslebens behinderter Menschen]. Zudem fällt bei Durchsicht dieser Publikationen auf, dass viele Stellungnahmen von Vertretern der Behindertenfürsorge stammen, jedoch kaum von Menschen mit Behinderung selbst. Zwar wird man aus dem Schweigen behinderter Menschen kaum Teilnahmslosigkeit oder Zustimmung gegenüber ihrer eigenen Situation ableiten können, zumal auch der beachtliche Zulauf bei Demonstrationen gegen das neue Gesetz (zuletzt im November 2009) auf eine gewisse Unzufriedenheit schließen lässt, ${ }^{10}$ und zudem zahlreiche Behinderte mit Unterstützung der Behindertenverbände Klage gegen das "Selbständigkeitsgesetz“ eingereicht haben. ${ }^{11}$ Eine politische und rechtliche Auseinandersetzung der Menschen mit Behinderung und der einzelnen Behindertenorganisationen mit den behaupteten Unzulänglichkeiten des Selbständigkeitsgesetzes findet also durchaus statt. Nichtsdestotrotz ist auffällig, dass die Wortführer in der mehr ideologisch geführten Diskussion um Behinderung und soziale Disparität beinahe ausschließlich aus den Reihen der Behindertenfürsorge stammen. Tatsächlich zeigt die Debatte, dass gerade die Verknüpfung von Behinderung und sozialer Disparität der Vermittlung von Vorstellungen und Zielen dient, die berufsspezifische Interessen und politische Ziele der Vertreter der Behindertenfürsorge widerspiegeln und nicht notwendig mit den Interessen der behinderten Menschen deckungsgleich sind.

Die Diskussion, die sich seit 2005 um Behinderung und soziale Disparität entfaltete, wird kaum kontrovers geführt, sondern ließ von Anfang an einen klaren Tenor erkennen: Alle Teilnehmer gehen wie selbstverständlich davon aus, dass Japan tatsächlich eine ungleiche Gesellschaft ist und behinderte Menschen Opfer dieser Ungleichheit sind, insofern sie durch Wettbewerb und soziale Kälte an den untersten Rand gedrückt werden. Zwar bekennen sich alle Autoren nach wie vor zu den Zielen der Normalisierung, insbesondere dem Recht auf Arbeit als allgemein menschliches Recht (z. B. Takahashi 2008: 126; Tada 2007: 15; Aoki 2006: 23). Das Selbständigkeitsgesetz wird jedoch einhellig als Rückzug (kōtai; vgl. Akamatsu 2006) des Staates aus seiner Schutzverantwortung verurteilt, durch den behinderte Menschen zunehmend in die soziale Iso- 
lation und Armut getrieben würden. Als Motive für diesen Rückzug werden die Tendenz zum „schlanken Staat“ und eine begleitende Einsparungspolitik sowie insgesamt eine menschenfeindliche Ökonomisierung des Denkens angenommen (Aoki 2006: 26; Tada 2007: 14; Takahashi 2008).

Als Instrumente der staatlichen Rückzugspolitik kritisieren die Vertreter der Behindertenfürsorge insbesondere zwei Elemente des neuen Systems: (1) die Belastung des behinderten Menschen mit einem Eigenanteil proportional $\mathrm{zu}$ seiner Inanspruchnahme von Pflegeleistungen (ōeki futan) sowie (2) die Forcierung regulärer Arbeit durch das Selbständigkeitsgesetz als Mittel zur Entlastung der Sozialkassen. Der nutzungsproportionale Eigenanteil, so die Kritiker, diskriminiere Menschen mit schwererer Behinderung gegenüber solchen mit leichteren und verschärfe die wirtschaftliche Ungleichheit (kakusa) zwischen diesen noch (Akamatsu 2006: 40). Zudem bestünde die Gefahr, dass behinderte Menschen zur Kostenvermeidung die Nutzung von Behinderteneinrichtungen einschränken würden und dadurch in die gesellschaftliche Isolation getrieben werden könnten. Dabei wird auf das soziale Phänomen des „Rückzugs aus der Gesellschaft“ (hikikomori oder tojikomori) Bezug genommen (Aoki 2006: 25; Saito 2007: 10). Hinsichtlich der Forcierung regulärer Arbeit beanstanden Kritiker insbesondere die Tatsache, dass behinderte Menschen für die Benutzung der Beschäftigungszentren, die den Übergang in das Erwerbsleben vorbereiten sollen, nun oft mehr zahlen müssten, als sie dort an Lohn erhielten. Dies würde wiederum zu der Abwärtsspirale beitragen, die viele Behinderte in die Armut führe (Akamatsu 2006: 41; Saitō 2007: 11). Konkrete Belege für diese Abwärtsbewegungen werden zwar selten genannt, jedoch wird auf die zahlreichen Fälle von „erzwungenen Doppelselbstmorden“ (muri shinjū) von behinderten Menschen seit 2005 hingewiesen, insbesondere auf den auch von den Medien vielbeachteten Fall einer Mutter, die die Tötung ihrer behinderten Tochter und den Selbstmordversuch mit ihrer Verzweiflung über die gestiegenen Pflegekosten begründete. ${ }^{12}$ In den meisten Fällen bleibt die konkrete Ursache jedoch offen.

An dieser Stelle sei angemerkt, dass die Behauptung einer Verarmung von behinderten Menschen lediglich drei Jahre nach Inkrafttreten des Selbständigkeitsgesetzes weder bestätigt noch falsifiziert werden kann. Aussagekräftige Statistiken liegen noch nicht vor. In jedem Fall würde eine solche Aussage ohnehin nur auf einen Teil der behinderten Menschen zutreffen, da deren wirtschaftliche Verhältnisse - wie beobachtet - bereits 2006 breit gestreut waren. Zudem wird man aus der Einführung des Eigenanteils allein kaum eine direkte Ursache für Verarmung ableiten können. Zwar zeigen Untersuchungen, dass dieser Anteil tatsächlich gestiegen ist (MHLW 2009), der Anteil kann jedoch auch ge- 
kürzt oder erlassen werden - eine Möglichkeit, von der auch ausgiebig Gebrauch gemacht wird (siehe etwa Saito [2007: 9] und Tada [2007: 14]; beide lehnen diese Maßnahmen jedoch als „Flickwerk“ ab). Auch das Problem der den Lohn übersteigenden Einrichtungsgebühren wird von manchen behinderten Menschen weniger als eine Frage des Geldes, denn als eine Frage der Ehre und Wertschätzung verstanden (siehe Saito [2007: 11]; vgl. auch Aoki [2006: 24]; Nakamura [2005: 58]).

Tatsächlich scheint die Sorge der Autoren für das Wohl behinderter Menschen häufig mit politischen und wirtschaftlichen Motiven verquickt zu sein. So ist - wie auch in der kakusa-Debatte allgemein - eine deutliche Opposition gegen den Geist des Neoliberalismus, mit dem die LDP unter Koizumi assoziiert wurde, zu verspüren. Eine radikale Kritikerin, selbst Angestellte in einer Behinderteneinrichtung, interpretiert das gegenwärtige Fürsorgesystem dementsprechend als eine zynische Einrichtung zur wirtschaftlichen Ausbeutung von behinderten Menschen.

Die Bestimmung der Selbständigkeit „entsprechend ihrer Fähigkeiten und Eignung" [Art. 1 SHSG] bedeutet einen radikalen Bruch zu den Vorstellungen von „Gleichheit" und „Normalisierung“ [...] Die Phrase „ungeachtet ihrer Behinderung" [ibid.] besagt, dass Menschen mit Behinderung nun eine Mitwirkungspflicht dazu haben, „zur Verwirklichung einer regionalen Gemeinschaft beizutragen, in der man ruhig und friedlich miteinander leben kann" [ibid.]. Besteht hier nicht die Gefahr, dass behinderte Menschen nach ihren Fähigkeiten unterschieden werden und Wohlfahrt als ordnungspolitische Maßnahme missbraucht wird? Das heißt man sagt: „Solche, die fähig sind $\mathrm{zu}$ arbeiten, machen wir zu arbeitenden Steuerzahlern“, „solche die nicht zur Verwirklichung einer regionalen Gemeinschaft beitragen, in der man ruhig und friedlich miteinander leben kann, schließen wir aus ordnungspolitischen Gründen aus und sperren sie weg" und ,diejenigen behinderten Menschen, die nicht arbeiten können und Pflege brauchen, machen wir zu Nutzern, die Pflegeleistungen einkaufen." (Takahashi 2008: 126)

Die meisten Autoren vermeiden solche Fundamentalangriffe und konzentrieren ihre Kritik konkret auf den nutzungsproportionalen Eigenanteil, den sie als Ausdruck eines falsch verstandenen, neoliberalen Selbstverantwortungsgedankens verstehen (Saito 2007: 11; Tada 2007; Suzuki 2005). Dieser sei für die Beurteilung der Umstände behinderter Menschen vollkommen unangemessen. Ein engagierter Journalist formuliert den Vorwurf folgendermaßen: „Wenn man von Geburt an behindert ist, ist das ,Selbstverantwortung'. Wenn man behindert ist infolge von Krankheit, ist es auch ,Selbstverantwortung'. Soll dieses Denken, das gegen- 
wärtig unser Land beherrscht, der Maßstab unseres Handelns sein?“ (Saito 2007: 11). Bemerkenswert an dieser Kritik ist zunächst ihr manipulativer Gehalt. Die rhetorische Frage nach der individuellen Verantwortlichkeit für Behinderung zielt auf ihre Verneinung und legt implizit die Alternative nahe, dass Behinderung allein der Solidargemeinschaft zu überantworten sei. So wird bereits eine Lanze für den Wohlfahrtsstaat gebrochen.

Bei näherer Betrachtung entpuppt sich dies jedoch als Scheinargument: In den meisten Fällen von Behinderung - wie auch bei menschlichem Unglück überhaupt - macht es keinen Sinn, die Frage nach der Verantwortlichkeit zu stellen, sondern allenfalls nach einer Lasten- und Risikoverteilung. Tatsächlich liegt die primäre Funktion des Eigenanteils in der finanziellen Lastenverteilung, nicht in der Zuschreibung einer wie auch immer gearteten Verantwortlichkeit. In Zeiten stetig knapper werdender Pflegeressourcen aufgrund des angesprochenen demographischen Wandels wird diese Funktion immer dringlicher, jedoch von den Autoren bemerkenswerterweise selten angesprochen. Dies mag seinen Grund darin haben, dass der wirtschaftliche Steuerungseffekt des Eigenanteils neben anderen marktwirtschaftlichen Elementen des neuen Systems wie z. B. der freien Wahl des Pflegedienstleisters - letztlich weniger zu Lasten des Leistungsempfängers als vielmehr mancher Pflegedienste geht (zur Parallele der Pflegeversicherung vgl. Arai [2003: 315]).

Dies führt zu den wirtschaftlichen Motiven des kakusa-Diskurses. Aus den Äußerungen der Autoren lässt sich - neben der Sorge um das Wohl der behinderten Menschen - eine deutliche Angst um die wirtschaftliche Lage der Pflegedienstleister herauslesen, in deren Brot die meisten Autoren stehen oder deren Interessen sie in Verbänden vertreten. So besteht die bereits angedeutete Befürchtung, dass behinderte Menschen weniger Dienste in Anspruch nehmen werden und insbesondere weniger häufig die Einrichtungen besuchen werden, die sie gewöhnlich besuchten. Die neu eingeführte tagesgenaue Berechnung der Nutzung dieser Einrichtungen führt dazu, dass sich ein Nutzungsrückgang unmittelbar in Einnahmeeinbußen widerspiegelt. Dies wiederum könnte zu Personalabbau und $\mathrm{zu}$ einer Verschlechterung der Arbeitsbedingungen in der Behindertenpflege führen (Akamatsu 2006: 42-44; Tada 2007: 15).

Dabei ist den Autoren bewusst, dass hier aufgrund der Einführung eines marktwirtschaftlichen Elements (Effizienzsteuerung und Wahlfreiheit des Dienstleisters) ein Interessenkonflikt zwischen behinderten Menschen und Pflegeanbietern entstehen kann: Es liege im Interesse der Anbieter, zur wirtschaftlichen Stabilisierung möglichst hohe Einnahmen zu erzielen; während es im Interesse der behinderten Menschen und ihrer Angehörigen sei, möglichst hohe Kosten zu vermeiden (Akamatsu 2006: 42-43; Saitō 2007: 9). Dass dieser Interessenkonflikt jedoch nicht allein 
auf die wirtschaftliche Ebene beschränkt bleibt, sondern - mehr oder weniger unbewusst - auch auf die Paradigmenebene durchschlägt, zeigt die folgende Klage eines Einrichtungsbetreibers über das Schicksal ehemaliger Nutzer seiner Einrichtung.

Einer hat sich wegen des Eigenanteils verzweifelt eine Arbeit gesucht. Anfänglich hat er in einer Spielzeugladenkette eines ausländischen Unternehmens die Ware einsortiert; jetzt muss er zum Beispiel schon Kunden bedienen. Andere haben aus lauter Not schließlich ihren Führerschein gemacht und sich auf Jobsuche begeben oder lassen sich auf einen Stock gestützt zwei Stunden in der vollen Bahn durchschütteln, um in die Fabrik fahren und dort im Akkord zu arbeiten.

Aber was sollen denn die Leute machen, die das nicht können? Ist das denn „Unterstützung“ zur „Selbständigkeit“, wenn man den Leuten einfach einen Tritt in den Hintern gibt und sagt, wenn Du nicht willst, dass man Dir Geld abnimmt, dann geh doch arbeiten?

Ich glaube, es gibt ganz verschiedene Formen der Selbständigkeit. In der freien Wirtschaft zu arbeiten mag ja Selbständigkeit bedeuten, aber in einer Behindertenwerkstatt mit Unterstützung zu arbeiten, sollte doch auch als Selbständigkeit gelten. (Zit. in Saitō 2007: 11)

Aus Sicht des Normalisierungsparadigmas ließen sich die genannten Beispiele von behinderten Menschen, die eine „normale“ Arbeit gefunden haben, sicherlich auch in einem positiveren Licht betrachten. Schließlich liegt es in dem Bestreben von Normalisierungsmaßnahmen, die Lebens- und Arbeitsverhältnisse so weit als möglich den durchschnittlichen Lebens- und Arbeitsverhältnissen anzugleichen. Das Mitleid des Einrichtungsbetreibers suggeriert jedoch, dass sich dieser eine weit weniger beschwerliche Normalität für behinderte Menschen wünscht, die nach wie vor in dem umhegten Raum der Behinderteneinrichtung stattfindet. Letztlich bedeutet dies eine Abkehr von den ursprünglichen Intentionen der Normalisierung sowie eine Rückwendung zu mehr Individualschutz und dem Paradigma des Wohlfahrtsstaates. Der wirtschaftliche Hintergrund und die gegenläufigen Interessen von behinderten Menschen und Betreibern von Pflegeeinrichtungen lassen vermuten, dass diese Umkehr nicht allein von Mitleid, sondern auch vom Interesse am Erhalt des eigenen wirtschaftlichen status quo der Einrichtungen motiviert sein könnte.

Dabei zeigen Untersuchungen des MHLW, dass von einem Abbau der gesetzlich vorgesehenen Behinderteneinrichtungen seit 2005 einstweilen nicht gesprochen werden kann (MHLW 2008b). Tatsächlich trifft der wirtschaftliche Umbau wohl vor allem die kleinen, privaten Behinderten- 
einrichtungen (shō-kibo sagyō-jo), die zahlenmäßig das staatliche Netzwerk um das Vierfache übertreffen und faktisch das Rückgrat der privaten (allerdings staatlich subventionierten) Behindertenpflege darstellten (Heyer 1999: 115). So ist es kein Zufall, dass alle der bisher zu Wort gekommenen Autoren in diesem privaten Netzwerk arbeiten oder deren Interessenvertretung, dem „Nationalen Netzwerk privater Behinderteneinrichtungen" (Kyosaren) angehören. Diese fürchtet nun, dass die meisten der kleinen Einrichtungen in dem neuen System in regionale Lebenshilfezentren (chiiki katsudō shien sentā) umgewandelt würden. Dies hätte allerdings zur Folge, dass die Einrichtungen staatliche Subventionen verlören und künftig der mehr oder minder prekären Finanzsituation der Kommunen ausgeliefert wären (Tada 2007: 14-15).

In diesem Licht gewinnen die Einlassungen der Autoren zur Existenzangst von behinderten Menschen in Japans kakusa-Gesellschaft eine andere Bedeutung: Sie verbindet sich mit der Existenzangst der kleinen Behinderteneinrichtungen, in dem unternehmerischen Wettbewerb des neuen Systems nicht mehr bestehen zu können beziehungsweise in den Strudel der finanziellen „Ungleichheit" zwischen den Gemeinden gerissen zu werden. So entpuppt sich der Diskurs um Behinderung und soziale Stratifikation auch als Klage eines Unternehmenssektors, der sich bereits als makegumi in dem neuen System der Behindertenfürsorge sieht.

\section{Schluss und Ausblick}

Der wirtschaftliche Subtext der Debatte führt uns zum Schluss an den Ausgangspunkt unserer Untersuchung zurück. So zeigt sich, dass die Debatte um Behinderung und soziale Ungleichheit in Japan seit 2005 mit Argumenten geführt wird, die sich vordergründig gegen ein neoliberales, utilitaristisches Leistungs- und Wettbewerbsdenken richtet, das allenfalls Menschen ohne Behinderung angemessen sei, Menschen mit Behinderung jedoch unbilligen Härten aussetzt. Tatsächlich dient der Protest jedoch zugleich auch dem Erhalt von kleinen Fürsorgeeinrichtungen, die sich nun - in Zeiten der Ressourcenknappheit - zunehmend selbst dem Wettbewerb und Leistungsdruck ausgesetzt fühlen. Der Wunsch nach mehr staatlichem Schutz gilt daher zunächst den Einrichtungen, schlägt sich jedoch tendenziell auch in einer Rückkehr zum Wohlfahrtsparadigma und dem Individualschutz von behinderten Menschen nieder, zu Lasten des Normalisierungsprinzips.

Dabei ist es leicht, diese Abkehr mit einer Kritik des Neoliberalismus zu verbinden, denn insofern Normalisierung den Übergang von mehr Schutz zu mehr Unterstützung bedeutet, ist dieses Konzept im Vergleich zum Wohlfahrtsprinzip tatsächlich als das „liberalere“, weniger paternalistische zu sehen. Während jedoch noch in Zeiten des relativen Wohl- 
stands die Einforderung des mit der Normalisierung verbundenen Rechts auf soziale Teilhabe im Vordergrund stand, zeigt sich nun - in Zeiten des demographischen Wandels und der Ressourcenknappheit - verstärkt auch die Beschwerlichkeit der Ausübung dieses Rechts. Die teilweise Abkehr von der Normalisierung erscheint in diesem Kontext daher zunächst als plausible Kritik an einem allein das Leistungsdenken betonenden und daher für Menschen mit Behinderung unwürdigen Gesellschaftsmodell. Allerdings stellt sich hier die Frage, ob die paternalistische Bemessung der noch zumutbaren Leistung nicht dem selben utilitaristischen Gesellschaftsmodell verhaftet ist, welches in der Kritik steht, und ob nicht stattdessen das Verhältnis von Leistung, Würde und Verteilungsgerechtigkeit gänzlich neu bestimmt werden müsste, um zu einem ausgewogenen Lösungsansatz zur Erreichung der Normalisierung zu gelangen (Nussbaum 2006; Sen 2009).

Abschließend sei angemerkt, dass die Behindertenverbände und Behindertenfürsorgeverbände mit ihren Protestaktionen im Jahre 2009 einen politischen Erfolg verbuchen konnten. So hat die neue Regierungspartei DPJ in ihrem Grundsatzpapier eine erneute Reform des Behindertenfürsorgesystems angekündigt, in der das Selbständigkeitsgesetz durch ein „Einheitliches Wohlfahrtsgesetz für Menschen mit Behinderung“ (Shōgaisha sōgō fukushi-hō, so der provisorische Titel) ersetzt werden soll. ${ }^{13}$ Als Eckpunkte dieses neuen Systems nennt das Manifest ein „lückenloses Versorgungssystem" und eine Rückkehr zur Eigenbeteiligung gemäß Leistungsfähigkeit. Die Ausarbeitung des Gesetzesentwurfs wird mit Rücksicht auf die noch ausstehende Ratifizierung der von Japan im Jahre 2007 unterzeichneten UN-Konvention über Rechte von Menschen mit Behinderung geschehen müssen. Auch die UN-Konvention verfolgt die Normalisierung als Fernziel. Wie die japanische Regierung dieses im Spannungsfeld partikularer Interessen sowie hinsichtlich der Möglichkeiten der Gesellschaft umsetzen wird, bleibt abzuwarten.

Urs Matthias Zachmann (U.Zachmann@1rz.uni-muenchen.de) is assistant professor at the Japan Centre, University of Munich (LMU). His fields of research are Japanese modern history and the sociology of law in contemporary Japan. He is author of China and Japan in the Late Meiji Period: China Policy and the Japanese Discourse on National Identity, 1895-1904 (London: Routledge, 2009).

\section{Anmerkungen}

* Ich danke den anonymen Gutachtern dieses Beitrags für ihre Hinweise und Anregungen.

1. Zur Diskussion der Konvention aus europäischer Sicht siehe Arnardottir und Quinn (2009) sowie Schulte (2009: 333-351). 
2. Eine Sammlung der wichtigsten, Menschen mit Behinderung betreffenden Gesetze findet sich im Internet auf der Homepage der DINF-Disability Information Resources (Shogai Hoken Fukushi Kenkya Joho Shisutemu) unter: http://www.dinf. ne.jp/doc/japanese/law/archives.html (Japanisch); und in Auszügen ins Englische übersetzt unter: http://www.dinf.ne.jp/doc/english/law/index.html.

3. Vgl. die Gesundheitsberichterstattung des Bundes unter: http://www.gbe-bund.de/ glossar/Behinderung_Statistik_der_schwerbehinderten_Menschen.html (letzter Zugriff 14.3.2010); zu der europäischen Situation siehe: http://ec.europa.eu/healtheu/my_health/people_with_disabilities/ (letzter Zugriff 14.3.2010).

4. Zur europäischen Situation vgl. Kommission der Europäischen Gemeinschaften (KOM 2007); zu Behindertenpolitik und Behindertenrecht in Europa vgl. Schulte (2009).

5. Das Konzept wurde ursprünglich für die soziale Rehabilitation von Menschen mit geistiger Behinderung entwickelt. In Japan sind insbesondere die Schriften Bengt Nirjes einflussreich (Nirje 2004 [1998], 2008).

6. Als prominentes Beispiel wäre hier die Verarbeitung des Themas Behinderung in dem TBS-Fernsehdrama „Beautiful Life“ (ausgestrahlt Januar-März 2000) zu nennen, das zeitweise eine Einschaltquote von mehr als $41 \%$ erreichte. Siehe hierzu: http://www.videor.co.jp/data/ratedata/junre/01drama.htm. Zur Unterrepräsentation von Menschen mit Behinderung in populären Serien, siehe Saito und Ishiyama (2005).

7. Die offizielle englische Übersetzung, die von dem Ministerium für Gesundheit, Arbeit und Wohlfahrt vorgeschlagen wird, lautet „Services and Supports for Persons with Disabilities Act“".

8. http://www.mhlw.go.jp/bunya/shougaihoken/service/aramashi.html.

9. Zur Integration von Menschen mit Behinderung im japanischen Bildungswesen und Arbeitsmarkt siehe auch Roeder (2001) und Meise (2000).

10. So berichtet NORMANET - Information Network of Persons with Disabilities, dass die Demonstrationen gegen das Selbständigkeitsgesetz im Oktober 2006 einen Zulauf von ca. 15000 Personen (http://www.normanet.ne.jp/ jadh/1031.html), im Oktober 2009 von ca. 10000 Personen hatten (http://www.normanet.ne.jp/ ictjd/ 091030.html) (letzter Zugriff 14. 3. 2010). Zu Protesten behinderter Menschen siehe auch Nakamura (2005: 58).

11. Zur Unterstützung und Koordination der Klagen hat sich beim Japan Council of Disability (Nihon Shogaisha Kyogikai) eine „Vereinigung zur erfolgreichen Durchsetzung der Klagen gegen das Selbständigkeitsgesetz" (Shogaisha jiritsu shien-ho sosho no shori o mezasu kai) gebildet.

12. Der Fall ereignete sich im März 2006 in Fukuoka. Das Gericht verwarf diese Begründung jedoch als haltlos (Saito 2007: 6).

13. Dieses Manifest von Juli 2009 findet sich unter: http://www.dpj.or.jp/special/ manifesto2009/txt/manifesto2009.txt.

\section{Literaturverzeichnis}

Akamatsu, Hidetomo. 2006. Hajimatta oeki futan: Shogaisha jiritsu shien-ho no genjitsu to kaizen no kadai [Der nutzungsproportionale Eigenanteil in Aktion: Die Realität des Gesetzes zur Unterstützung der Selbständigkeit von Menschen mit Behinderung und die Aufgabe seiner Verbesserung]. Jinken to Buraku Mondai 750. 38-48.

Aoki, Osamu. 2006. Kakusa shakai to "shogai“ o motsu kodomolkatei no seikatsu: Nomaraizeshon no shinka no saki [Die Ungleiche Gesellschaft und das Leben von Kindern und Familien mit „Behinderung“: Das Ziel der Intensivierung von Normalisierung]. Minna no Negai 473. 23-26. 
Arai, Makoto. 2002. The aging society and the social security system in Japan. In Harald Conrad \& Ralph Lützeler (eds.), Aging and social policy: A German-Japanese comparison, 37-49. Munich: Iudicium.

Arai, Makoto. 2003. Landesbericht Japan. In Bernd von Maydell, Rainer Pitschas \& Bernd Schulte (eds.), Behinderung in Asien und Europa im Politik- und Rechtsvergleich, 303-342. Baden-Baden: Nomos.

Arai, Makoto. 2009. Japan's welfare system for people with disabilities. In Bernd von Maydell, Rainer Pitschas, Peter Pörtner \& Bernd Schulte (eds.), Politik und Recht für Menschen mit Behinderung in Europa und Asien, 389-397. Baden-Baden: Nomos.

Arnardottir, Oddny Mjoll \& Gerard Quinn (eds.). 2009. The UN convention on the rights of persons with disabilities: European and Scandinavian perspectives. Leiden \& Boston: Martinus Nijhoff.

Conrad, Harald. 2001. The Japanese social security system in transition: An evaluation of current pension reforms. München: Iudicium.

Coulmas, Florian, Harald Conrad, Annette Schad-Seifert \& Gabriele Vogt (eds.). 2008. The demographic challenge: A handbook about Japan. Leiden: Brill.

Finkelstein, Victor. 1980. Attitudes and disabled people: Issues for discussion. New York: International Exchange of Information in Rehabilitation.

Hara, Junsuke \& Kazuo Seiyama. 2005. Inequality amid affluence: Social stratification in Japan. Melbourne: Trans Pacific Press.

Hashimoto, Kenji. 2003. Class structure in contemporary Japan. Melbourne: Trans Pacific Press.

Heyer, Katharina. 1999. Between equality and difference: The politics of disability in Japan. Japanstudien 11. 105-133.

Heyer, Katharina. 2000. From welfare to rights: Japanese disability law. Asia-Pacific Law and Policy Journal 1 (1). http://www.hawaii.edu/aplpj/articles/APLPJ_01.1_ heyer.pdf (letzter Zugriff 14. 3. 2010).

Itoh, Makoto. 2008. Japan: eine Neubewertung des Neoliberalismus. In Ingo Schmidt (ed.), Spielarten des Neoliberalismus: USA, Brasilien, Frankreich, Deutschland, Italien, Indien, China, Südkorea, Japan, 236-253. Hamburg: VSA.

KOM (Kommission der Europäischen Gemeinschaften). 2007. Situation von Menschen mit Behinderungen in der Europäischen Union: Europäischer Aktionsplan 2008-2009. http://eur-lex.europa.eu/LexUriServ/LexUriServ.do?uri=COM:2007: 0738:FIN:DE:PDF (letzter Zugriff 14. 3. 2010).

Meise, Sabine. 2000. Normalization is best - Einstellung japanischer Lehrer zur Integration behinderter Schüler in das Regelschulsystem. Die neue Sonderschule 45(5). $352-362$.

MHLW (Kōseirōdōshō). 2005. Shōgaisha jiritsu shien-hō ni tsuite [Über das Gesetz zur Förderung der Selbständigkeit behinderter Menschen]. http://www.mhlw.go.jp/ bunya/shougaihoken/jiritsushienhou01/ (letzter Zugriff 14. 3. 2010).

MHLW. 2007a. „Fukushi kara koyo e“ suishin gokanen keikaku: Sanko shiryo [Der Fünfjahresplan zur Förderung der Tendenz „Von der Wohlfahrt zur Beschäftigung": Referenzmaterial]. http://www.kantei.go.jp/jp/singi/seichou2/dai4/siryou4. pdf (letzter Zugriff 14. 3. 2010).

MHLW. 2007b. Heisei 18-nendo kōchin (chingin) getsugaku ni tsuite [Über den Monatsverdienst in Behinderteinrichtungen 2006]. http://www.mhlw.go.jp/houdou/ 2007/10/h1031-4.html (letzter Zugriff 14. 3. 2010).

MHLW. 2008a. Heisei 18-nen shintai shōgaiji / -sha jittai chōsa kekka [Untersuchungsergebnisse zur Situation von körperlich behinderten Kindern/Erwachsenen im Jahre 2006]. http://www.mhlw.go.jp/toukei/saikin/hw/shintai/06/ (letzter Zugriff 14. 3. 2010). 
MHLW 2008b. Heisei 20-nen shogai fukushi sabisu nado keiei jittai chosa kekka [Untersuchungsergebnisse zur Unternehmenssituation der Wohlfahrtsdienste für Menschen mit Behinderung im Jahre 2008]. http://www.mhlw.go.jp/houdou/2008/11/ h1120-3.html (letzter Zugriff 14. 3. 2010).

MHLW. 2009. Shogaisha jiritsu shien-ho no shiko zengo ni okeru riyo-sha no futan nado ni kakaru jittai chosa kekka ni tsuite [Untersuchungsergebnisse bzgl. der Kostentragungslast von Nutzern vor und nach Durchführung des SJSH]. http://www. mhlw.go.jp/stf/houdou/2r98520000002sre-img/2r98520000002ssw.pdf (letzter Zugriff 14. 3. 2010).

Motozawa, Miyoko. 2009. Behinderung und Familie in Japan. In Bernd von Maydell, Rainer Pitschas, Peter Pörtner \& Bernd Schulte (eds.), Politik und Recht für Menschen mit Behinderung in Europa und Asien, 399-410. Baden-Baden: Nomos.

Nagai, Michio. 1954. Herbert Spencer in early Japan. Far Eastern Quarterly 14 (1). $55-64$.

Naikaku-fu. 2007. Shogaisha hakusho Heisei 19-nen-ban [Weißbuch für Menschen mit Behinderung 2007]. Tokyo: Naikaku-fu.

Naikaku-fu. 2009. Shogaisha hakusho Heisei 21-nen-ban [Weißbuch für Menschen mit Behinderung 2009]. Tokyo: Naikaku-fu.

Nakamura, Karen. 2005. Severe disabilities, liberalism, and social welfare policy in Japan and the United States. Anthropology News 46 (9). 58. http://www.disabilitystudies.jp/nakamura/publications/2005-AN-DisabilityJapan/ AN-DisabilityInJapan.pdf (letzter Zugriff 14. 3. 2010).

Nirje, Bengt. 2004 [1998]. Nomaraizeshon no genri: Fuhenka to shakai henkaku o motomete [Das Prinzip der Normalisierung: Auf der Suche nach Universalisierung und sozialer Reform], 3. Aufl. Tokyo: Gendai Shokan.

Nirje, Bengt. 2008. Saiko Nomaraizeshon no genri: Sono hirogari to gendai-teki igi [Erneute Überlegungen zum Normalisierungsprinzip: Seine Verbreitung und Bedeutung in der Gegenwart]. Tokyo: Gendai Shokan.

Nussbaum, Martha Craven. 2006. Frontiers of justice: Disability, nationality, species membership. Cambridge, MA: Belknap Press of Harvard University Press.

Rawls, John. 1971. A theory of justice. Cambridge, MA: Belknap Press of Harvard University Press.

Rawls, John. 1996. Political liberalism. New York: Columbia University Press.

Roeder, Maike. 2001. Behinderte Menschen in Japan: Eine Studie zur schulischen Bildung und beruflichen Integration. Bonn: Bier'sche Verlagsanstalt.

Saitō, Shinichi \& Reiko Ishiyama. 2005. The invisible minority: Under-representation of people with disabilities in prime-time TV dramas in Japan. Disability \& Society 20(4). 437-451.

Saitō, Takao. 2007. „Kakusa shakai“ ni okeru shōgaisha jiritsu shien-hō no jitsuzō [Das wahre Antlitz des Gesetzes zur Unterstützung der Selbständigkeit von Menschen mit Behinderung in der „ungleichen Gesellschaft“]. Minna no Negai 479. $6-11$

Schulte, Bernd. 2009. Behindertenpolitik und Behindertenrecht in Europa: Behindertenpolitische und behindertenrechtliche Grundlegung: Die Europäische Union. In Bernd von Maydell, Rainer Pitschas, Peter Pörtner \& Bernd Schulte (eds.), Politik und Recht für Menschen mit Behinderung in Europa und Asien, 305-364. BadenBaden: Nomos.

Sen, Amartya. 2009. The idea of justice. Cambridge, MA: Belknap Press of Harvard University Press.

Shakai Hoken-chō. 2009. Heisei 19-nen-do shakai hoken jigyō no gaikyō [Allgemeine Situation der Sozialversicherungsarbeit im Jahre 2007] http://www.sia.go.jp/infom/ tokei/gaikyo2007/gaikyo.pdf (letzter Zugriff 14. 3. 2010). 
Stein, Mark S. 2006. Distributive justice and disability: Utilitarianism against egalitarianism. New Haven, CT: Yale University Press.

Suzuki, Tsutomu. 2005. Oeki futan-shugi no ideorogt-sei: Byodo gensoku e no chosen [Das Ideologische an dem Prinzip des nutzungspropotionalen Eigenanteils: Eine Kampfansage an das Gleicheitsprinzip]. In Shogaisha Seikatsu Shien Shisutemu Kenkyn-kai (ed.), Shogaisha jiritsu shien-ho to oeki futan: Kore o fukushi to yoberu $k a$ [Das Selbständigkeitsgesetz und der nutzungsproportionale Eigenanteil: Kann man das noch Wohlfahrt nennen?], 75-88. Kyoto: Kamogawa Shuppan.

Tachibanaki, Toshiaki. 2006. Kakusa shakai: Nani ga mondai nano ka [Die ungleiche Gesellschaft: Wo liegt das Problem?]. Tokyo: Iwanami Shoten.

Tada, Kaoru. 2007. Jiko sekinin-ron o haikei to shita „oeki futan“ wa najimanai [Ich kann mich nicht mit dem „nutzungsproportionalen Eigenanteil“" im Kontext der Selbstverantwortungsdebatte abfinden]. Minna no Negai 479. 13-15.

Takahashi, Michiko. 2008. Kakusalhinkon shakai to shogaisha no jiritsu shien [Die Ungleichheits-/Armutsgesellschaft und die Unterstützung der Selbständigkeit von Menschen mit Behinderung]. In Makino Tomio \& Eigo Murakami (eds.), Kakusa to hinkon ga wakaru 20-ko [20 Vorträge zum Verständis von sozialer Ungleichheit und Armut], 120-131. Tokyo: Akashi Shoten.

Takizawa, Hitohiro. 2006. Shogaisha-kan kakusa no hoteki kenkya: Kakusa honin to jiritsu shien [Eine juristische Untersuchung der Ungleichheit zwischen behinderten Menschen: Die rechtliche Anerkenntnis von Ungleicheit und Unterstützung der Selbständigkeit]. Kyoto: Minerva Shobō.

Traphagan, John W. 2007. Moral discourse and old-age disability in Japan. In Benedicte Ingstad \& Susan Reynolds Whyte (eds.), Disability in local and global worlds, 259-286. Berkeley: University of California Press.

Wolfensberger, Wolf. 1972. The principle of normalization in human services. Toronto: National Institute on Mental Retardation. 